\title{
Effect of Ecofriendly Microencapsulated Textile Products In Joint Pain and Aesthematic Respondents
}

\author{
Devinder Kaur and Neelam Grewal \\ Clothing and Textiles, Punjab Agricultural University, Ludhiana, Punjab
}

\begin{abstract}
The study was conducted to develop aromtheraputic textile products for joint pains/ asthma. Fabric treated with eco-friendly microcapsules of eucalyptus, jasmine, lemon and pine oil respectively were used for the treatment of respondents. Knee caps, gloves, Pillow covers and handkerchieves were made joint \& ashtetic respectively for the respondents out of cotton fabric. Respondents suffering were undergone for the wear trail of two months. To get the results through interview schedule data collected. The results showed that the developed products had a positive effect on the emotional state of the respondents (87\%), improved in quality of night sleep $(95 \%)$, out door activities (67\%) and reduced medicine in take (50\%). The results also showed soothing effect on the skin of the respondents after using these aromatherapic textiles.

Therefore, it was recommended that the aromatherapic textile products made with eco-friendly chemicals like betacyclodextrine as binder and chitosin as sheath along with different essential oils in core can be used for developing products. These products retain their fragrance for at least 20 washings if" washed under controlled conditions.
\end{abstract}

Key words: Aromatherapic, Betacyclodextrin, Essential oils, Microencapsulation, Optimization, Products, Textiles.

\section{Introduction}

The role of the textile finisher has become increasingly demanding and now requires a careful balance between the compatibility of different finishing products and treatments as well as the application processes used to provide textiles with desirable properties. Growing trends and escalating standards leave no stone unturned to boost the current status of textile industry. Aroma finishing of textiles is one such immaculate magnanimous entry into the textile culture.

An increasingly popular application of the microencapsulation technique is the use of fragrance encapsulated capsules onto fabrics. The capsule that can be applied on the fabrics resist breaking under normal conditions. This fragrance from the capsule is released only during the application of pressure onto the fabric and acts as a healing substance which is used in aromatherapy (Sudha et al 2005).

Aromatherapy is a medicinal technique that helps people to heal with natural substances, by using pure fragrance compound and essential oils. This type of healing with the help of fragrances by using essential oils has been found to have an effect on feelings, moods and emotions. To make this aromatherapy easier, textiles can be chosen as medium since they are close friends humans. The end-use product includes ribbons, handkerchiefs, curtains and furnishings

(Harini et al 2007)

In the textile and garment world, finishes play a vital role for quality value. The textile consumers become much more aware and desire greater safety and better comfort by avoiding unpleasant odour. Chitosan is a popular finishing agent of cotton fabrics used to make microcapsules. It is eco friendly and? give good effect to human skin in addition its finishing properties. It is an analogous of cellulose with $\mathrm{N}$-acetyl groups instead of hydroxyl groups in position $\mathrm{C} 2$ modification. Chitin is converted into chitosan by alkali treatment. Alkali splits $\mathrm{N}$-Acetyl groups into free amino groups $(75-95 \%)$ that provide fungi static and bacteriostatic effects. It is chemically called as beta- $(1,4)-2$ (amino)-2-deoxy-D-glycopranose. In aqueous solution at $\mathrm{pH}<6.5$, it is its protonated form and behaves like a cationic polyelectrolyte and interacting with negatively charged molecules. In addition to its antimicrobial effect, it is eco friendly and has a good effect on human skin. It can be applied by microencapsulation or by reactive bonding to cellulose and by cross linking of chitosan. The chitosan finish gives high absorbency, moisture control, non-allergic, non-toxic and biodegradable properties to textiles (Jeyakodi et al 2008).

B-Cyclodextrine is another popular eco-friendly finishing agent that can be strongly embedded into the fabric. Even after machine wash, these remain active. B-cyclodextrin does not cause skin irritation, sensitization and does not have mutagenic effects. These are non-reducing, cyclically linked oligosaccharides produced by certain micro organisms of cultivated starch, that are capable of forming inclusion compounds with molecules that fit into their cone-shaped hydrophobic cavity. As a result of the inclusion, the physico-chemical properties of the compounds are changed, e.g. the vapour pressure of volatile substance is reduced, and stability against light or air is enhanced. On the other hand, the harmful and unpleasant odour in the surrounding may be eliminated. Furthermore, $\beta$-cyclodextrins clamped on cellulose do not affect its properties, and at the same time retain their 
ability

with the help of medical specialists of prestigious hospitals of Ludhiana city. Wear trails were conducted on a total of 40 respondents. The respondents were made to use the developed articles for 2 months and their responses regarding the effect of these products were studied with the help of a structured interview schedule. However, washing fastness other properties intensity of the developed products were studied in the laboratory.

\section{Results And Discussion}

ß-cyclodextrin was used as binder of microcapsules on to the to form inclusion complexes with other suitable molecules. Thus, fabric. According to Reuscher et al (2008) ß-cyclodextrin

ß-cyclodextrins are the first choice in preparing aromatherapy textiles (Harini et al 2007).

Concentrated essential oils are far more valuable to humankind than just having pleasing aromas. The healing properties of these essential oils were known in the ancient times, from Egypt to India and China. On rubbing, these oils are absorbed through the skin, leaving no toxins as these are excreted from the body through urine, feces, perspiration and exhalation. The essential oils have a proven history of treating many ailments like acne, burns, insomnia, depression, indigestion, dandruff, cervical cancer, etc. (Foster 2006). Therefore, the present investigation has been planned with the following objectives in mind:

1. To develop utility articles using cotton fabrics treated with contains reactive groups in the ring which help in building blocks as cross linking agents to incorporate favorable properties permanently on the surface of cotton, whereas the oils are the main sources of aroma. Therefore, the concentration of both these were optimized by studying the effect of these on selected physical properties of the substrate.

Optimization of concentration of ß-cyclodextrin

Optimization was done to arrive at the optimum concentration for giving the $\beta$-cyclodextrin treatment to the fabric. Effect of 8, 10 and 12 per cent concentrations on fabric thickness, tensile strength, bending length, crease recovery, and flexural rigidity of the substrate was studied. microcapsules of aromatic essential oils and eco-friendly

Table 1 Optimization of $\beta$-cyclodextrin concentration for chemicals

\begin{tabular}{|c|c|c|c|c|c|}
\hline $\begin{array}{l}\text { Percent } \\
\text { Conc } \\
\text { entration }\end{array}$ & $\begin{array}{c}\text { Fibre } \\
\text { thicknes } \\
\mathrm{s}(\mathrm{mm})\end{array}$ & $\begin{array}{l}\text { Tensile } \\
\text { strength } \\
\text { (1.af) } \\
\text { Warp Weft }\end{array}$ & 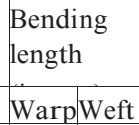 & $\begin{array}{l}\text { Crease } \\
\text { recovery } \\
\text { Warp Wefin }\end{array}$ & $\begin{array}{l}\text { Overall } \\
\text { flexural } \\
\text { rigidity }\end{array}$ \\
\hline Control & 15.40 & \begin{tabular}{|l|l|}
56.05 & 57.28 \\
\end{tabular} & $4.16 \quad 4.11$ & $95.33 \quad 100.00$ & 76.47 \\
\hline 8 & $16.60 *$ & $45.94 * 51.32 *$ & $* 3.23 * 3.25 *$ & * $90.44 * 93.66 *$ & $69.88 *$ \\
\hline 10 & 19.40 & \begin{tabular}{|l|l|}
59.01 & 60.87 \\
\end{tabular} & $\begin{array}{ll}4.45 & 4.26 \\
\end{array}$ & \begin{tabular}{|l|l|}
96.00 & 104.66 \\
\end{tabular} & 77.99 \\
\hline 12 & 21.00 & 46.1952 .00 & $\begin{array}{ll}3.95 & 3.78 \\
\end{array}$ & 91.3394 .66 & 72.65 \\
\hline CD 5\% & 1.23 & \begin{tabular}{l|l}
6.54 & 4.21 \\
\end{tabular} & \begin{tabular}{l|l}
0.18 & 0.23 \\
\end{tabular} & \begin{tabular}{l|l}
6.76 & 7.47 \\
\end{tabular} & 1.989 \\
\hline
\end{tabular}

2 To undertake wear trails and effect of evaluate the developed products on the respondents.

\section{Materials And Methods}

The present study was conducted to optimize the conditions for preparing microcapsules and then applying on to the fabric to develop aromatherapic products. Later to evaluate the effect on respondents through wear trials of the developed products .

Four different essential oils, i.e. lemon, pine, jasmine and eucalyptus were selected for two different health conditions, i.e. asthma and joint pains respondents. For optimization of conditions for preparing microcapsules three concentrations of oils were selected, i.e. 25,35 and 45 per cent on the bases of sensorial evaluation and physical properties. Ecofriendly chemicals were used for development and application of microcapsules on the woven and knitted cotton fabrics. Chitosan was used as a binder since chitosan polymers (due to the acetyl- amino groups) can be used as biodegradable sheath material for microencapsulation and result in controlled release of essential oils for therapeutic purposes.

For the application of fragrant microencapsules to cotton $\beta$-cyclodextrin was used as it does not cause skin irritation, skin sensitization and does not have mutagenic effect. Further, these are capable of forming inclusion compound with molecules. Further more, $\beta$-cyclodextrin clamped on cellulose do not affect the cellulosic properties and it keeps their ability to form

microencapsulation on the basis of effect on various physical

properties of treated fabric

* Significant at 5 per cent

The results pertaining to the effect of different concentrations of - cyclodextrin, i.e. 8,10 and 12 per cent on selected physical properties of the test specimens have been furnished in table 1. It is evident from the table that there was no marked change in the physical properties due to finishing treatment at 10 per cent level. 
The fibre thickness of the control sample was $15.40 \mathrm{~mm}$ but increased to 16.60 when 8 per cent -cyclodextrin was applied. A further increase to 19.40 and 21.00 was observed at 10 and 12 per cent concentration of cyclodextrin, respectively. These readings which were significantly different from control sample.

The tensile strength of the sample treated with 10 per cent - cyclodextrin was maximum and was at par with the control sample. The bending length of the control sample was 4.16 in the warp and 4.11 in the weft direction. It was found that an increase the concentration of -cyclodextrin up to a certain level, the inclusions complexes with other suitable molecules.

Three

bending length also increased.

concentrations of $ß$-cyclodextrin were also selected, i.e. 8,10 and

12 per cent for the finish application along with microcapsules made of essential oils and chitosan, Kaur D (2009).

These developed products were then distributed to the respondent

The data showed that the crease recovery angle was maximum in the case of 10 per cent concentration, i.e. 96.00 in the warp direction and 104.66 in the weft direction, being very close to control specimens. The overall flexural rigidity was found maximum in the case of 10 per cent concentration of - cyclodextrin, i.e. 77.99 closely followed by (76.47) control specimen.

The results showed that for fabric thickness, tensile strength, bending length, crease recovery, moisture absorption and flexural rigidity properties, 10 per cent concentration of -cyclodextrin did not have marked effect on the properties of the substrates and are in line with the findings of Wang et al (2007) who reported that cyclodextrin does not have a marked effect on its physical properties of the substrates and is ideal for preparing inclusion compounds for microencapsulation of essential oils.

Effect of developed products on emotional state of respondents

Figure 1 Distribution of subjects according to the effect of develoed

products on emotional state of the respondents

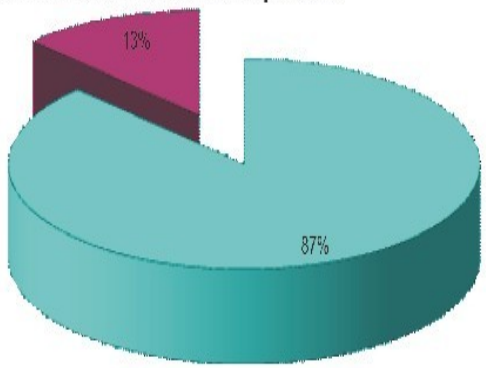

QAffect the emoticnal state $\square$ Notaffeciec the emotions

The effect of the developed products on respondents was studied in terms of effect on emotional state, sense of wellbeing, type of effect and any allergic reaction to these products. It is evident from Fig. 1 that these products had an effect on the emotional state of the total of 87 per cent respondents

The results are in line with those reported by Herz (1998) who found that the scented, fabrics influence the emotional state and well being, by enhancing mood, increasing creative thinking, relaxing or stimulating and facilitate creative thinking.

Effect of developed products on night sleep and outdoor activities of the respondent

Figure 2 Effect of developed aromatherapic products on night sleep of the respondent

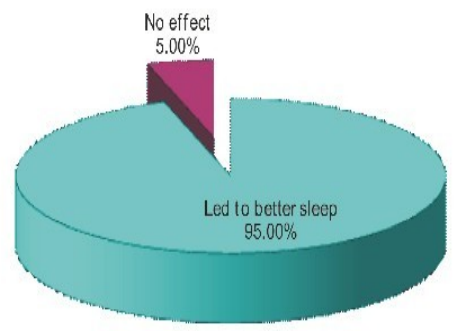

QLed to better sleep

aNoeffect 
An enquiry into the effect of developed products on night sleep of the respondents revealed in Fig. 2 that there was an effect on the quality of night sleep of 95 per cent of the total respondents. Wang et al (2005) also found that patient suffering high blood pressure felt sedative effects when they used a pillow made of fabric treated with lavender, basil, lemon microcapsules.

\section{Figure 3 Effect of aromatherapic products on outdoor activities of the respondents}

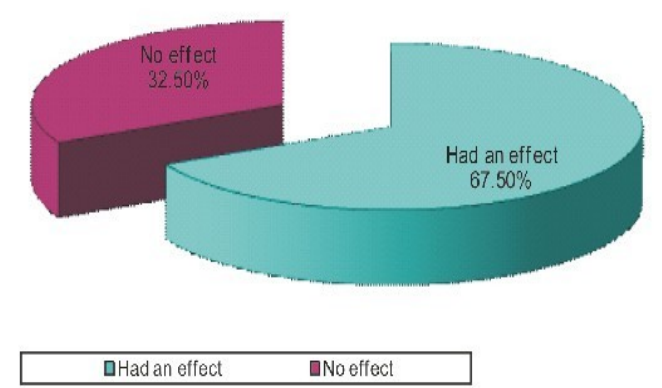

Fig. 3 shows the effect of the aromatherapic products on the outdoor activities of the respondents. A total of 67.50 per cent respondents reported an increase in outdoor activities with the use of aromatherapic products

Effect of developed product on medicine intake of the respondents

Figure 4 Effect of developed aromatherapic products on medicine intake of the respondents

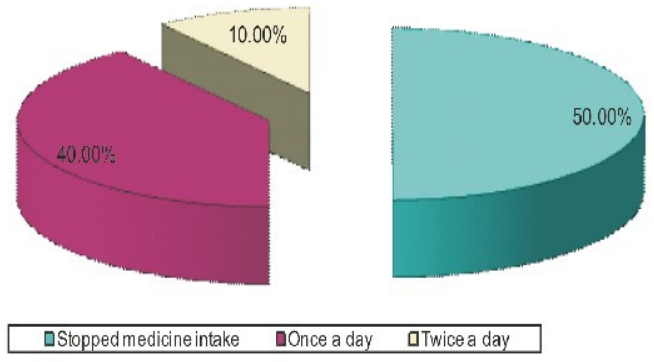

It can be seen in Fig. 4 that 50 per cent of the total respondents, 10 (50\%) from each category, stopped taking medicines all together

Washing fastness of the developed products

Life of the developed products or the effectiveness of finish in holding the fragrance was studied in the form of washing fastness of the treated fabric after 20 wash cycle of lounder-o- meter. The data revealed that the products could withstand to 20 lounder-o-meter washings. The life could be further improved if washed only with cold water without using soap.

While studying the overall rating of the developed products it was found that the most of the respondents were ready to buy aromatherapic products 'for the sake of health' and due to reduction in the intake of medicines. Thus, it can be envisaged that the aromatherapic products are acceptable to a large majority of the respondents as these gave relief. Further more, if available, the respondents were ready to buy these products for the sake of better health.

Wang et al (2005) also found that aromatherapic textiles are the first choice for people who wanted to be healthy in their daily life. The researchers also found that fabrics treated with different perfumes helped in creating a "cocooning" environment among the respondents led to the therapic effect of these products.

\section{Conclusions}

Microcapsules of 10 per cent concentration of betacyclodextrin as a finishing material and 45 per cent core material of lemon, pine, jasmine or eucalyptus essential oils, using chitosan (sheath material) can be application on cotton fabric for the development of the textile products. 
The developed products had a positive effect on the emotional state of the respondents $(87 \%)$, improved in quality of night sleep (95\%), out door activities (67\%) and reduced medicine in take (50\%).

Impact of 20 launder-o-meter washings on the intensity of fragrance of the oils revealed that the pine oil hold the maximum fragrance, followed jasmine and eucalyptus oil. Lemon oil showed minimum intensity rating of 1.90 after 20 washes.

\section{References}

[1]. Foster, G. 2006. Understanding aromatherapy.www.gabrielfoster.com.

[2]. Harini, K. Leena, A. and Giri Dev, V. R. 2007. Microencapsulation and its application. Synthetic Fibres XIII 6 21-28.

[3]. Herz, R. S. 1998. Are odors the best cues to memory? A cross- modal comparison of associative memory stimuli. Annals of the New York Academy of Sciences 855. (Original not seen cited by Pierce, J. Tovia, F. and Weathers, N. 2005, 2006. Scent-inflused Textile to Enhance Consumer Experiences, NTC Project No. F05-PH03. www.wacker.com).

[4]. Jeyakodi, M. and Ammayappamn, L. 2008. Enhancement of effect of aloe vera for its antimicrobial propetiges on cotton and protein fibers using selected additives. www.fibre2 fashion.com.

[5]. Kaur, D. 2009. Development and Evaluation of Microencapsulated aromatheraptic Textile Products. Dissertation submitted at PAU Ludhiana.

[6]. Reuscher, H. and Hirsenkom, R. 2008. BETA W7 MCT - Newways in surface modification. Wacker-Chemie GmbH, Hams - SeidalPlatz4, D-81737 Munchen, GERMANY.

[7]. Sudha, S. Devgiri, V. R. and Neelkanda, R.2005. Microencaptulation:an overview :25-27.www.@google.com.

[8]. Wang, C. X. and Chen, Sh. L. 2007. Aromachology and its application in the textile field, fibres and textiles in Eastern Europe 13(6): 41-44.www.@google.com.

[9]. Wang, C.V. and Shen She, L. 2007. Aromachology and its Application in the textile field, fibers and textiles in Eastern Europe 13(6): $41-44$. 